\title{
On-axis Transmission Kikuchi Diffraction for Orientation Mapping of Nanocrystalline Materials in the SEM
}

\author{
E. Brodu ${ }^{1,2}$, E. Bouzy ${ }^{1,2}$ and J-J. Fundenberger ${ }^{1,2}$ \\ 1. Laboratoire d'Etude des Microstructures et de Mécanique des Matériaux (LEM3), UMR CNRS 7239, \\ Université de Lorraine, Metz, France \\ 2. Laboratory of Excellence on Design of Alloy Metals for low-MAss Structures (DAMAS), University \\ of Lorraine, Metz, France
}

Electron Backscatter Diffraction (EBSD) in the SEM is routinely and widely used to produce local orientation maps of the microstructure of materials, via the automated recording and analysis of Kikuchi diffraction patterns. Orientation maps can then be easily processed to extract the following information: phase distribution, grain size distribution, orientation of individual grains, disorientation inside grains and grain boundary characterization. EBSD is thus overall a very powerful technique.

One of the limits of EBSD though is its lateral resolution. Indeed, with a lateral resolution of about 50 $\mathrm{nm}$ [1], many features remain out of reach. Precisely with the objective of overcoming this limitation, a new diffraction technique was recently developed in the SEM, referred to as TKD or t-EBSD [2]. TKD (Transmission Kikuchi Diffraction) makes use of the same hardware as EBSD. The difference is that TKD uses electron transparent samples, prepared the same way as for TEM, instead of polished- bulk materials as for EBSD. The Kikuchi diffraction patterns in TKD are thus produced by the electron transmitted through the thin sample, instead of by the backscattered ones as for EBSD. It leads to a reduction of interaction volume and consequently an improvement in lateral resolution, from about 50 $\mathrm{nm}$ for EBSD to less than $10 \mathrm{~nm}$ for TKD [3-5].

Following the recent developments of TKD, an even newer configuration was developed at LEM3: onaxis TKD [6]. This technique does involve a hardware modification in comparison to the conventional off-axis TKD first proposed by Keller and Geiss [2]. The scintillator is moved from its vertical position to a horizontal position under the transparent sample. The scintillator thus sits in the direction of highest transmitted intensity, enabling much faster acquisition speeds. It was previously estimated that on-axis TKD is 20 times faster than conventional TKD [7]. With a faster acquisition speed, on-axis TKD will be more suitable than conventional TKD for the analysis of beam sensitive materials. It also makes on-axis TKD less prone to beam drifts and instabilities.

In term of lateral resolution, on-axis TKD falls seemingly in the same range as off-axis TKD (i.e. below $10 \mathrm{~nm}$ ). However, the strength of on-axis TKD is that even the thinnest samples are exploitable (below $50 \mathrm{~nm}$ ), which is where the interaction volume will be the smallest and consequently the resolution the best. This should give the edge to on-axis TKD in term of lateral resolution. Indeed, with very thin samples, most of the transmitted intensity is confined to a small angular range, especially with low-Z materials. The consequence is that little signal reaches the detector of the conventional configuration $[3,4]$, while the on-axis detector beneficiates from an intense signal. A comparison between the two techniques remains to be done. In the meantime, the map displayed in figure 1 shows that grains as small as $10 \mathrm{~nm}$ are perfectly resolved with on-axis TKD, in particular in the top part of the map, where the sample is the thinnest. Twins of only 2-4 pixels wide are resolved as well, corresponding to 6-12 $\mathrm{nm}$. Also, the indexation rate is $90 \%$, with grain boundaries presenting a remarkably low fraction of pixels 
not indexed. The effective resolution corresponds here roughly to one or twice the pixel size, i.e. 3-6 nm. Indeed, most grain boundaries present a lateral noise with an amplitude within twice the pixel size. The physical resolution on the other hand is around 6-9 nanometers for this particular experiment, as evaluated from the distance between two unrelated diffraction patterns across grain boundaries.

\section{References:}

[1] DR Steinmetz, S Zaefferer, Materials Science and Technology 26 (2010) p.640.

[2] R Keller, RH Geiss, Journal of Microscopy 245 (2012) p.245.

[3] R van Bremen et al, Ultramicroscopy 160 (2016) p.256.

[4] YZ Wang et al, Journal of Microscopy 264 (2016) p.34.

[5] W Trimby, Ultramicroscopy 12 (2012) p.16.

[6] J-J Fundenberger et al, Ultramicroscopy 161 (2016) p.17.

[7] H Yuan et al, Journal of microscopy - Accepted 2016

[8] B Beausir, J-J Fundenberger, ATOM - Analysis Tools for Orientation Maps, http://atom-software.eu/

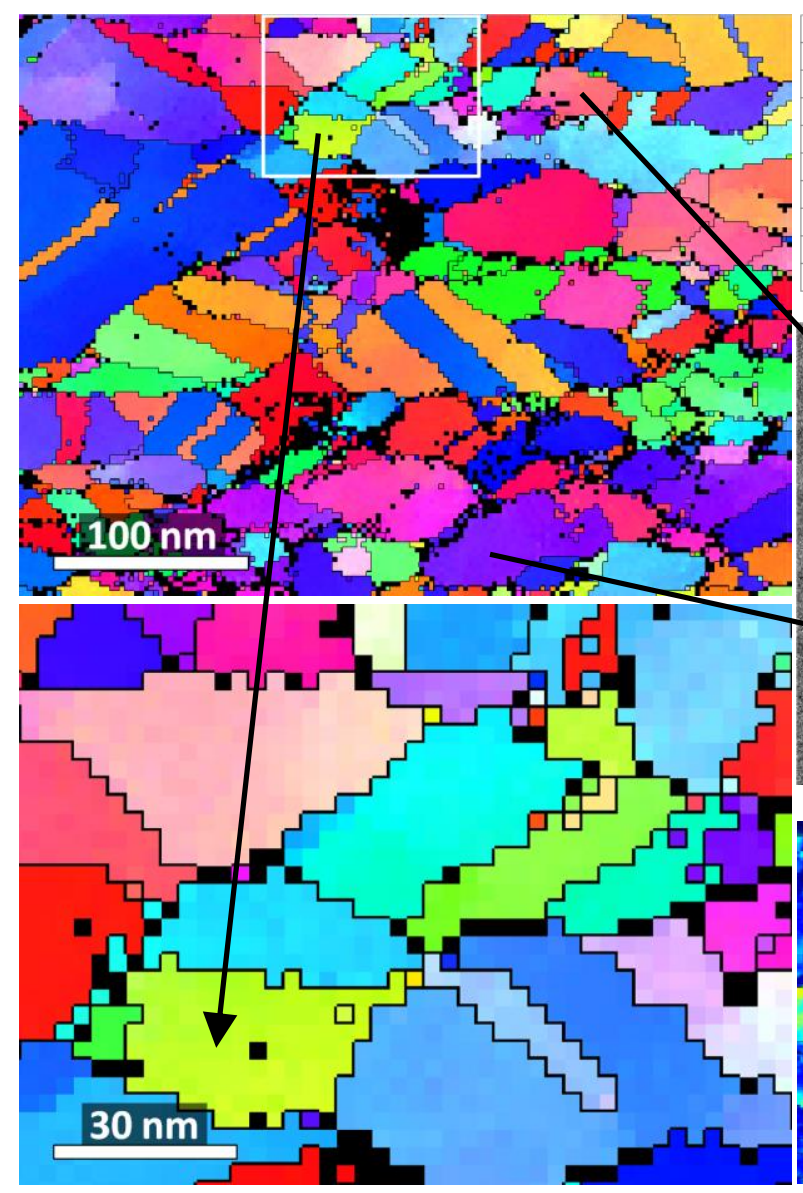

\begin{tabular}{|l|l|l|l|}
\hline Microscope & Zeiss Supra 40 & Acquisition soft. & Brucker Esprit 2.1 \\
\hline Configuration & on-axis TKD & Pattern resolution & $400 \times 400$ pixels \\
\hline Camera & Brucker e-Flash1000 & Hough resolution & 60 pixels \\
\hline Detector & Brucker OPTIMUS & Integration time & $15 \mathrm{~ms} \times 2$ images $(30 \mathrm{~ms}$ total) \\
\hline Accelerating voltage & $30 \mathrm{keV}$ & Acquisition time & $9 \mathrm{~min} 50 \mathrm{~s}$ \\
\hline Aperture & $60 \mu \mathrm{m}-\mathrm{HC}(\approx 2.5 \mathrm{nA})$ & Map size & $160 \times 120$ pixels \\
\hline Working distance & $7.1 \mathrm{~mm}$ & Step size & $2.9 \mathrm{~nm}$ \\
\hline Sample tilt & $0^{\circ}$ & Indexation rate & $90 \%$ \\
\hline Magnification & $243 \mathrm{kx}$ & Material & Ni, pulsed electrodeposition \\
\hline Detector-sample distance & $19.7 \mathrm{~mm}$ & Sample thickness & Unknown (below $100 \mathrm{~nm})$ \\
\hline
\end{tabular}

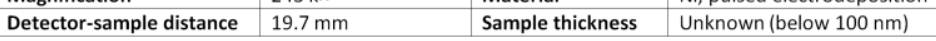

Figure 1. Top left: Raw orientation map obtained by on-axis TKD. Top right: Experimental parameters. Bottom left: Close up of the map. Middle right: typical diffraction patterns. The left and right patterns correspond to the thinnest and thickest part of the map respectively. Bottom right: Internal disorientation of grains with a scale bar of $3^{\circ}$ (left) and grain boundary characterization over the quality pattern map (right) with red boundaries for $\left[58-62^{\circ}\right]$, white for $\left[10-58^{\circ}\right]$ and finally black for $\left[2-10^{\circ}\right]$ with the ATOM software [8] 